\title{
BMJ Open Identifying psychosocial characteristics that predict outcome to the UPLIFT programme for people with persistent back pain: protocol for a prospective cohort study
}

\author{
Hayley Thomson, ${ }^{1,2}$ Kerrie Evans, ${ }^{\circledR 3,4,5}$ Jonathon Dearness, ${ }^{1}$ John Kelley, ${ }^{1}$ \\ Kylie Conway, ${ }^{1}$ Collette Morris, ${ }^{1}$ Leanne Bisset, ${ }^{4,6}$ Gwendolijne Scholten-Peeters, ${ }^{\bullet} 7$ \\ Pim Cuijpers, ${ }^{\oplus 8}$ Michel W Coppieters ${ }^{\oplus 6,7}$
}

To cite: Thomson H, Evans K, Dearness J, et al. Identifying psychosocial characteristics that predict outcome to the UPLIFT programme for people with persistent back pain: protocol for a prospective cohort study. BMJ Open 2019;9:e028747. doi:10.1136/ bmjopen-2018-028747

- Prepublication history for this paper is available online. To view these files, please visit the journal online (http://dx.doi. org/10.1136/bmjopen-2018028747).

Received 21 December 2018 Revised 24 April 2019

Accepted 21 June 2019
Check for updates

(C) Author(s) (or their employer(s)) 2019. Re-use permitted under CC BY-NC. No commercial re-use. See rights and permissions. Published by BMJ.

For numbered affiliations see end of article.

Correspondence to

Professor Michel W Coppieters; m.coppieters@griffith.edu.au

\section{ABSTRACT}

Introduction Prognostic screening of people with low back pain (LBP) improves utilisation of primary healthcare resources. Whether this also applies to secondary healthcare remains unclear. Therefore, this study aims to develop prognostic models to determine at baseline which patients with persistent LBP are likely to have a good and poor outcome to a 5-week programme of combined education and exercise ('UPLIFT') delivered in a secondary healthcare setting.

Methods and analysis A prospective cohort study of 246 people with persistent LBP will be conducted in a secondary healthcare outpatient setting. Patients will be recruited from a physiotherapy-led neurosurgical screening clinic. Demographic data, medical history and psychosocial characteristics will be recorded at baseline. Fear avoidance beliefs, pain self-efficacy, LBP treatment beliefs, pain catastrophising, perceived injustice, depression, anxiety and stress, disability level, pain intensity and interference, health status and social connectedness will be considered as potential prognostic variables, which will be assessed using selfreported questionnaires. Participants will attend the UPLIFT programme, consisting of weekly 90 min group sessions that combine interactive education sessions and a graded exercise programme. The outcome measure to identify good and poor outcome is the Global Rating of Change scale, assessed at completion of the UPLIFT programme and at 6 months follow-up. Multiple imputation analyses will be performed for missing values. Prognostic models will be developed using multivariable logistic regression analyses, with bootstrapping techniques for internal validation. We will calculate the explained variance of the models and the area under the receiver operating characteristic curve. Furthermore, we will determine whether participation in the UPLIFT programme is associated with changes in psychosocial characteristics.

Ethics and dissemination Gold Coast Health Service Human Research Ethics Committee (HREC/18/QGC/41) and the Griffith University Human Research Ethics Committee (GU Ref No: 2018/408) approved the study. Dissemination
Strengths and limitations of this study

- The results of this study may help identify factors that influence outcome for patients with low back pain in secondary healthcare settings.

- The sample size allows assessment of a broad spectrum of psychosocial predictor variables, but does not allow the reassessment of previously studied biological variables.

- This pragmatic study evaluates existing best evidence-informed clinical practice.

- This study is a valuable first step in identifying potential predictors or effect modifiers, but without a comparison group we cannot guarantee that the predictors identify those who do well with the UPLIFT programme, or those that have a favourable natural history.

- Patients are recruited from one hospital which may limit the generalisability of the findings.

of findings will occur via peer-reviewed publications and conference presentations.

Trial registration number ACTRN12618001525279.

\section{INTRODUCTION}

Low back pain (LBP) is the leading cause of disability worldwide ${ }^{12}$ and the population burden continues to increase globally. ${ }^{3}$ In 2015, the global point prevalence of activity-limiting LBP was $7.3 \%$, implying that 540 million people were affected at any one time. ${ }^{1}$ General practitioners in Australia refer patients with persistent LBP to a medical specialist at a rate of 5.2 per 100 patients with $\mathrm{LBP}^{45}$ despite specialist intervention being indicated for only a small proportion of these patients. ${ }^{6}$

Clinical guidelines recommend a biopsychosocial management approach for people 
with persistent LBP. ${ }^{78}$ High-quality evidence supports the use of biopsychosocial interventions, focusing on active management strategies that address psychosocial domains and improvements in physical function. ${ }^{79}$ Despite this increasingly accepted approach, optimal management for people with persistent LBP remains a source of contention in the literature ${ }^{910}$ with few established interventions demonstrating long-term effectiveness. ${ }^{11}$

While biopsychosocial interventions are promising, experts suggest that patient outcomes could be improved with stratified care approaches, matching subgroups of patients to interventions from which patients are most likely to benefit. ${ }^{12}$ Certainly, in primary healthcare, stratified care using prognostic tools can identify patients with persistent LBP likely to respond to specific interventions, improve patient outcomes and reduce healthcare costs. ${ }^{14-16}$ To date, there is no evidence to suggest that these findings can be translated into secondary or tertiary healthcare settings. ${ }^{417}$

Clinical guidelines recommend secondary care referral for patients with LBP when treatment needs are too complex for primary healthcare management. ${ }^{17}{ }^{18} \mathrm{~A}$ recent review suggested that the use of prognostic tools validated in primary healthcare to direct treatment decisions for patients with LBP are not helpful to direct treatment in secondary healthcare. The review highlighted that investigations of factors that influence responders in secondary healthcare are significantly lacking. ${ }^{4}$

The vast majority of research pertaining to patients with persistent LBP in primary healthcare settings has identified that psychosocial factors, rather than biological factors, are more likely to identify responders to specific interventions. Factors such as distress and anxiety, ${ }^{11}$ fear avoidance beliefs, ${ }^{12}$ pain self-efficacy, ${ }^{13}$ body perception, ${ }^{14}$ pain catastrophising ${ }^{15}$ and perceived injustice ${ }^{16}$ have been shown to predict responses to specific interventions. Therefore, programmes aimed at also addressing these factors may be more effective than programmes addressing biological factors only.

The UPLIFT programme is an innovative evidence-informed biopsychosocial group intervention for patients with persistent LBP who have been referred to a neurosurgical screening clinic but for whom surgery is not indicated. A retrospective analysis of the first 120 patients who completed the programme revealed a success rate of $55 \%$, based on a clinically significant improvement in the Global Rating of Change (GROC) score obtained at completion of the programme.

We hypothesise that utilisation of health service resources may be further improved if good and poor outcome to the UPLIFT programme could be identified accurately and objectively at baseline. Referral into the programme is currently dependent on the clinical opinion of the screening physiotherapists. There is recognition that these decisions may be improved with the addition of a tool to help identify those patients likely to gain the most benefit from the UPLIFT programme and, conversely, those patients who are at risk of a poor outcome who may require alternative treatment approaches.

The primary aim of the proposed study is to derive prognostic models from baseline variables from the psychosocial domains to identify good and poor outcome to the UPLIFT programme. We hypothesise that the derived prognostic models will be significantly better at predicting good and poor outcome than the current $55 \%-45 \%$ ratio for treatment success versus non-success. The secondary aim is to gain insight into what psychosocial factors change following completion of the UPLIFT programme.

\section{METHODS AND ANALYSIS \\ Study design and setting}

The study is a single-centre, prospective cohort study with 6 months follow-up (figure 1). Participant recruitment will take place at the outpatient department of Gold Coast University Hospital, Australia. Data collection started in July 2018 and we anticipate data collection will be completed by December 2019. All participants will provide written informed consent before participating in the trial. The study will be implemented and reported in line with the Strengthening the Reporting of Observational Studies in Epidemiology and Transparent Reporting of a multivariable prediction model for Individual Prognosis or Diagnosis (TRIPOD) statements.

\section{Participants}

The physiotherapy-led neurosurgical screening clinic at the hospital triages patients with persistent pain. According to their needs and possible benefits, people with persistent LBP may be referred to various management pathways, such as surgical consultation (ie, neurosurgery), specialist consultation (ie, neurology, rheumatology), further technical investigations, individual physiotherapy or group physiotherapy (ie, the UPLIFT programme) and/or other individual allied health interventions (ie, psychology, dietetics and pharmacy). Patients with persistent LBP will be recruited from those patients referred to the UPLIFT programme. Inclusion criteria are: (1) adults over 18 years, (2) persistent LBP (ie, at least 3 months), (3) sufficient English reading and writing skills to complete questionnaires and to comprehend and participate in the interactive education sessions within the programme. Exclusion criteria are: (1) contraindications for exercise, (2) recent spinal surgery (within preceding 12 months), (3) active inflammatory conditions, such as rheumatoid arthritis and (4) neurological conditions.

\section{The UPLIFT programme}

UPLIFT is a 5-week programme consisting of weekly 90 min group sessions that combine education and exercise. Each education session targets a different theme. Themes are: (1) pain neuroscience, (2) activity pacing, (3) flare-up management, (4) acceptance and (5) adopting 
People with persistent back pain referred by local GPs to the Physiotherapy-led Neurosurgical Screening Clinic at the hospital

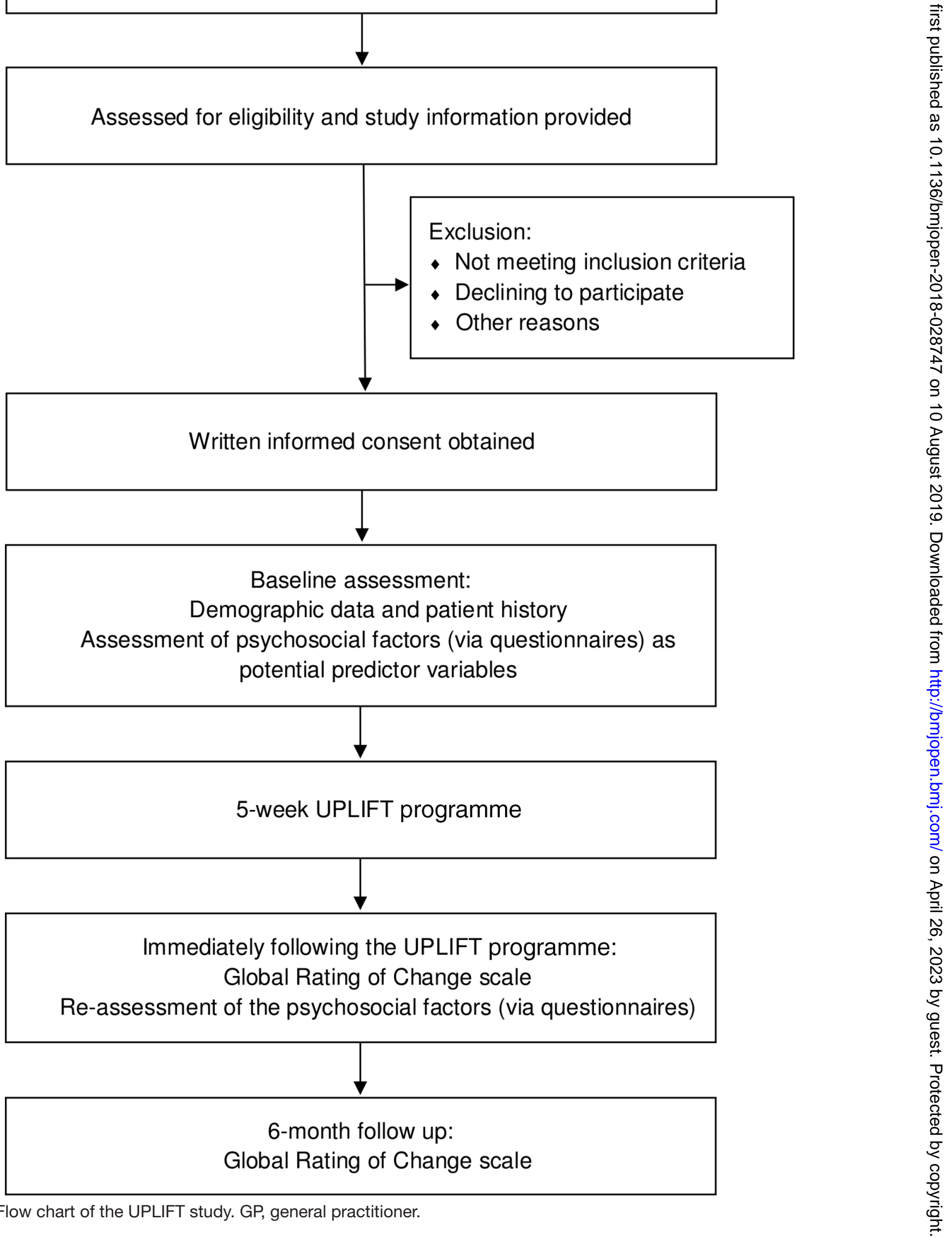

Figure 1 Flow chart of the UPLIFT study. GP, general practitioner. 
healthy lifestyle behaviours (including, sleep hygiene and meaningful movement). UPLIFT incorporates motivational interviewing techniques allowing participants to undertake appropriate cognitive and experiential processing of the programme's content. Volunteer peer mentors who have successfully completed UPLIFT assist in facilitating the programme by sharing their own experience of the UPLIFT programme. The mentors are given 3 hours of face-to-face training by the programme's lead physiotherapists. The interactive delivery style of UPLIFT aims to improve participants' knowledge, which underpins true conceptual and behaviour change. ${ }^{19}{ }^{20}$ Patients in the UPLIFT programme are considered active partners in their rehabilitation, aiming to reconceptualise their understanding of pain, develop active coping strategies to self-manage LBP and re-engage in value-based activities. The combination of education and physical activity aims to reduce fear of movement and provide each participant with guidance and direction towards active behaviour change.

Recommended physical treatments, particularly for persistent LBP, include a graded activity or exercise programme that targets improvements in function and prevention of worsening disability. ${ }^{7}$ There is no evidence demonstrating superiority of one form of exercise over another for people with LBP. ${ }^{21} 22$ Therefore, guidelines recommend exercise programmes take an individual's needs, preferences and capabilities into account when deciding the type of exercise most appropriate for that person. ${ }^{73}$ The exercise component of the UPLIFT programme incorporates safe, graduated exposure to feared movement and graded conditioning exercises based on a patient's individual goals. ${ }^{23}$ The five themes from the education component of the programme are consolidated during each exercise session.

A week-by-week overview of the UPLIFT curriculum is provided in table 1 .

\section{Predictor variables}

Evidence does suggest that psychosocial domains such as pain catastrophising, self-efficacy, patient expectations and beliefs are more predictive of patient outcomes than changes in biomechanical and structural targets of therapy. ${ }^{245}$ There is a lack of knowledge about prognostic factors that influence recovery in people with persistent LBP in secondary healthcare. ${ }^{46}$ It is also unclear whether these factors will be different from prognostic factors derived from primary healthcare. Models using biomedical predictor variables in a postsurgical lumbar discectomy population have also demonstrated poorly explained variance. ${ }^{27}$ With persistent LBP causing significant personal suffering and distress, impacting on daily function, as well as impairing social and occupational engagement, ${ }^{1}$ potential predictor variables from psychosocial domains are the focus of this study. We have not included previously proven predictors such as previous episodes of back pain, presence of leg pain, body mass, smoking, physical activity, education, employment status, comorbidities and work satisfaction. ${ }^{1}$ When choosing the outcome domains of interest, we considered both the target population, and the nature of the intervention (the UPLIFT programme).

The potential predictor variables are: (1) fear avoidance; (2) pain self-efficacy; (3) LBP treatment beliefs; (4) pain catastrophising; (5) perceived injustice; (6) depression anxiety and stress; (7) disability level; (8) pain intensity and interference; (9) self-reported health status and (10) social connectedness.

\section{Fear avoidance}

Fear avoidance is assessed by the Fear Avoidance Beliefs Questionnaire (FABQ). ${ }^{28}$ The FABQ measures fear of pain and subsequent avoidance of physical activity due to fear. The FABQ consists of 16 items divided into two subscales: fear avoidant beliefs about work and physical activity. Items are scored on a 6-point Likert scale, ranging from 'completely disagree' to 'completely agree'. Higher scores on the FABQ indicate greater fear and avoidance beliefs. The FABQ has good test-retest reliability. ${ }^{28}$ The FABQ is a preferred questionnaire to assess fear avoidance. ${ }^{29}$

\section{Pain self-efficacy}

The Pain Self-Efficacy Questionnaire (PSEQ) ${ }^{30}$ measures the confidence people with pain have to perform activities while in pain. Low self-efficacy is a predictor of being at risk of long-term disability and depression, while higher self-efficacy appears to enhance and maintain the long-term effects of rehabilitation. The PSEQ is a 10-item questionnaire. Items are scored on a 6-point Likert scale, ranging from 'not at all confident' to 'completely confident'. Scores are summed with a maximum score of 60 , with higher scores indicating better pain self-efficacy. The scale has high test-retest reliability and construct validity. ${ }^{30}$ The PSEQ is a preferred questionnaire to assess pain self-efficacy. ${ }^{29}$

\section{Treatment beliefs}

Treatment beliefs are assessed by the Low Back Pain Treatment Beliefs Questionnaire (LBP-TBQ). ${ }^{31}$ The LBP-TBQ assesses treatment beliefs in people with LBP and investigates how these beliefs affect treatment uptake and adherence. The LBP-TBQ has four subscales (pain medication, exercise, manual therapy and acupuncture), comprising 16 items each. Items are scored on a 5-point Likert scale, ranging from 'strongly disagree' to 'strongly agree'. Scores are summed, with a maximum score of 80 in each subscale. Higher scores indicate more positive beliefs about the efficacy of specific LBP treatments. The scale demonstrates good homogeneity and good convergent and discriminant validity. ${ }^{31}$

\section{Pain catastrophising}

The Pain Catastrophising Scale (PCS) ${ }^{32}$ measures the degree of catastrophic thinking related to the individual's pain condition, which can affect pain experiences. The PCS consists of 13 items with three subscales: 


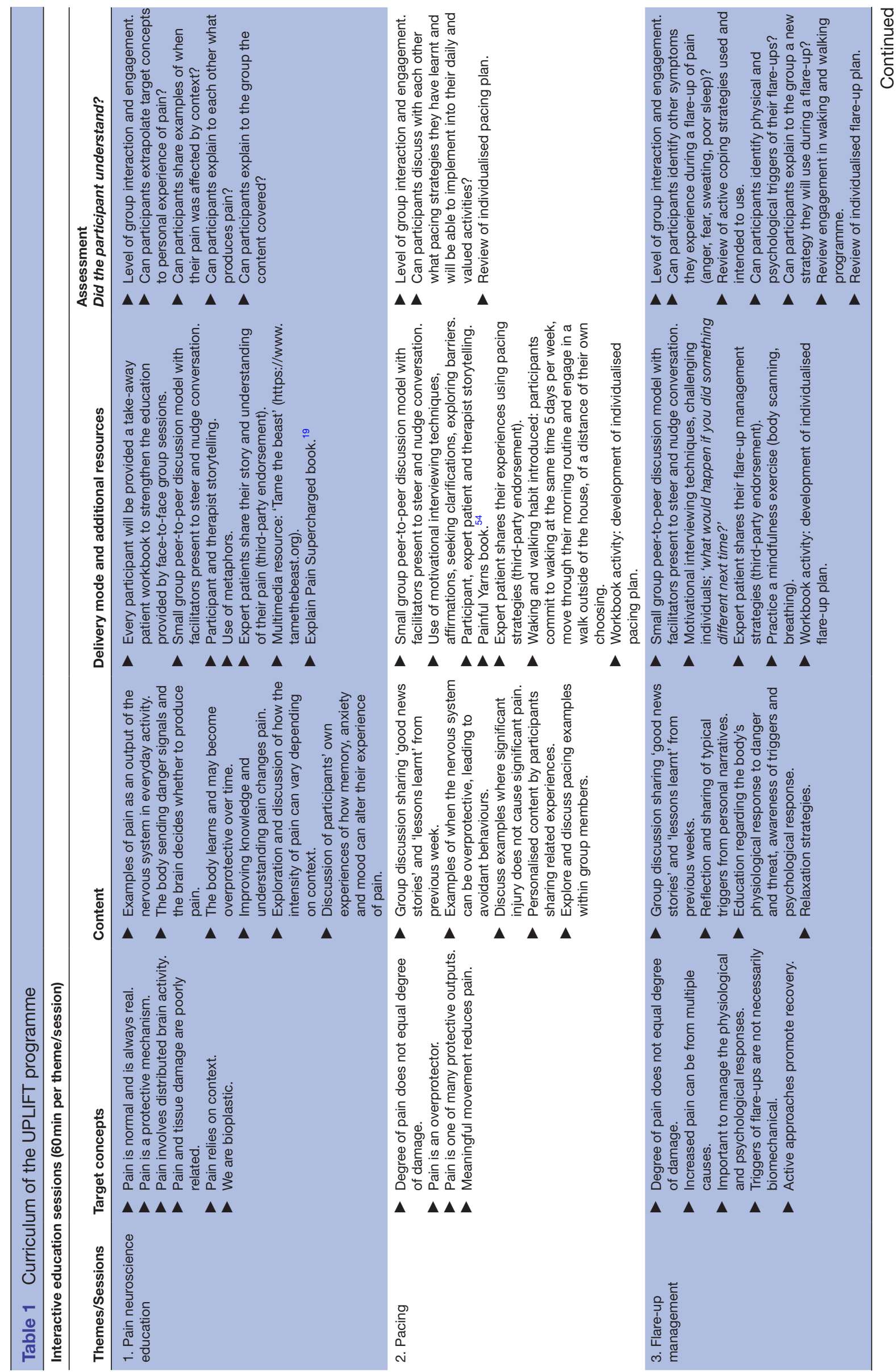



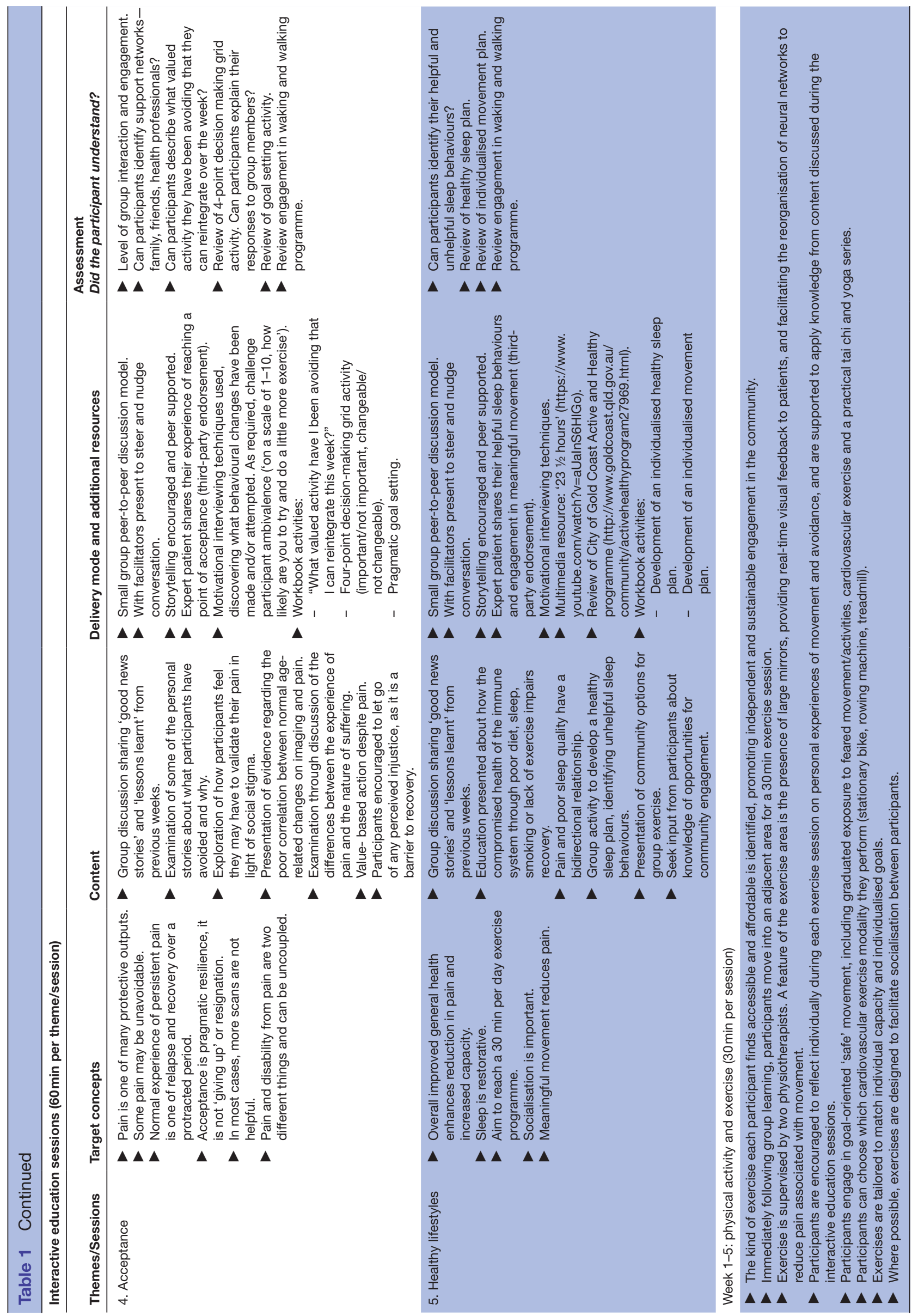
magnification, rumination and helplessness. Responses are scored on a 5-point Likert scale, ranging from 'not at all' to 'all the time'. Scores above 30 indicate a clinically relevant level of pain catastrophising. The PCS has adequate test-retest reliability. ${ }^{33} 34$ The PCS is a preferred questionnaire to assess pain catastrophising. ${ }^{29}$

\section{Perceived injustice}

Perceived injustice is assessed using the Injustice Experience Questionnaire (IEQ). ${ }^{32}$ The IEQ measures the degree to which individuals perceive their postinjury life as being characterised by injustice. Respondents indicate how frequently they think of 12 statements. Responses are scored on a 5-point Likert scale ranging from 'not at all' to 'all the time'. Higher scores indicate higher perceived injustice. The IEQ has high test-retest reliability and good construct validity. ${ }^{32}$

\section{Depression, anxiety and stress}

The Depression Anxiety and Stress Scale 21 (DASS-21) ${ }^{35}$ is a short form of the original DASS-42 instrument. The scale measures symptoms of negative emotional states (depression, anxiety and stress) experienced during the last week. The DASS-21 comprises 21 items, with responses scored on a 4-point Likert scale ranging from 'did not apply to me at all', to 'applied to me very much, or most of the time'. The three subscales each have individual cut-off scores for severity of emotional state. For depression, scores from 11 to 13 indicate a 'severe' level of depressive symptoms. For anxiety, scores from 8 to 9 indicate a 'severe' level of anxiety symptoms. For stress, scores from 13 to 16 indicate 'severe' level of stress symptoms. The DASS-21 has adequate reliability, and strong convergent validity. ${ }^{35}$ The depression subscale of the DASS-21 is a preferred questionnaire to assess depression. ${ }^{36}$

\section{Disability level}

Disability level is assessed by the Oswestry Disability Index (ODI). ${ }^{37}$ The ODI measures the level of function (degree of disability) in activities of daily living for a person with LBP. The ODI consists of 10 questions with responses rated on a 6-point Likert scale reflecting the degree of limitation for each activity, ranging from no limitation to maximum limitation. Scores are converted to a percentage of degree of disability. The ODI has high testretest reliability and construct validity. ${ }^{37} 38$

\section{Pain intensity and interference}

The Brief Pain Inventory (BPI ${ }^{39}$ measures two different aspects of pain: pain intensity and interference in everyday life. The BPI is a 9-item questionnaire. Respondents first indicate their area(s) of pain on a body chart, then answer items on the two subscales. Responses on both subscales are scored on an 11-point Likert scale ranging from 'no pain' to 'pain as bad as you can imagine' for pain intensity, and 'does not interfere' to 'completely interferes' for interference. The BPI has good test-retest reliability and construct validity. 3940

\section{Self-reported health status}

Self-reported health status is assessed by the Short Form Health Survey (SF-36). ${ }^{41}$ The measure consists of 36 items covering eight domains (bodily pain, physical functioning, role limitations due to physical health problems, role limitations due to personal or emotional problems, emotional well-being, energy/fatigue, social functioning and general health perceptions). Higher scores indicate better self-reported health. The SF-36 has good test-retest reliability. ${ }^{41-43}$

\section{Social connectedness}

The Social Connectedness Scale $(\mathrm{SCS})^{44}$ measures the degree to which a person feels connected to others in their social environment. The SCS focuses on the emotional distance or connectedness between the self and other people. It is an 8-item questionnaire with scores recorded on a 6-point Likert scale ranging from 'strongly agree' to 'strongly disagree'. Higher scores indicate stronger perceived social connectedness. The scale has good testretest reliability and high construct validity. ${ }^{44}$

\section{Outcome measure}

\section{Global Rating of Change}

The primary outcome to determine success of the UPLIFT programme is the score on the 11-point GROC scale, ranging from -5 ('very much worse') to +5 ('completely recovered'). A score of $\geq+3$ will define success. GROC scores will be assessed immediately following completion of the programme and at 6 months follow-up, with the latter being the primary time-point. The GROC scale is recommended for use in clinical research as a core outcome measure of global improvement with treatment ${ }^{45}$ and is responsive to change. ${ }^{46}$

\section{Procedure}

Patients with persistent LBP will be recruited and assessed for eligibility from those patients referred to the UPLIFT programme via the physiotherapy-led neurosurgical screening clinic. Eligible patients will receive oral and written information about the study and provide written consent prior to participating.

Once consent is provided, participants will complete the Adult Pre-Exercise Screening Tool ${ }^{47}$ to screen for risk factors associated with exercise and will also complete the Patient Initial Referral Questionnaire (Electronic Persistent Pain Outcomes Collaboration 2011). This form includes personal details (such as demographic data and medical history), work status, medication use and also the BPI, DASS-21, PSEQ and PCS questionnaires). Participants will then complete the other questionnaires (FABQ, LBP-TBQ, IEQ, ODI, SF-36 and SCS). Collection of all baseline data takes between 30 and $45 \mathrm{~min}$ via paper survey and occurs under the supervision of a research assistant at the university teaching hospital. Participants will then commence the 5-week UPLIFT programme. Immediately on completion of the programme, participants will complete the same questionnaires as those used 
at baseline as well as the GROC scale. Six months after completing the UPLIFT programme, participants will be sent the GROC scale by the research assistant, either electronically or a printed copy, depending on patient preference. This will be completed at home without staff assistance. All baseline measures will be collected by a researcher blinded to programme participation. Assessment at baseline and on immediate completion of the programme will occur at the university teaching hospital.

\section{Sample size estimation}

Considering (a) the anticipated success rate of $55 \%$ based on retrospective analysis of the first 120 participants in the UPLIFT programme, (b) consideration of 10 potential predictor variables (see below) for each multivariable prognostic model (separate models will be developed to identify good and poor outcome to the UPLIFT programme) and (c) the rule-of-thumb of 10 participants in the limiting sample size per predictor variable, 223 participants are required to prevent overfitting of the model ( 10 potential predictor variables $\times 10$ events per variable equals 100 participants in the limiting sample size (ie, the least frequent outcome which is $45 \%$ ); for 100 participants with a non-favourable outcome (45\%), 123 participants will have a favourable outcome $(55 \%)$; hence, a sample of 223 participants is needed). Considering an anticipated drop-out rate of $10 \%$ at 6 months follow-up (primary time-point), a sample size of 246 participants is required. This sample size is comparable with other prognostic studies in musculoskeletal research. ${ }^{48-50}$

As enrolling in the study does not involve a substantial extra burden to the participants of UPLIFT, we anticipate (based on pilot data) that the majority of patients referred to the UPLIFT programme will consent to enrol in the study.

\section{Statistical analysis}

Data integrity and storage

All data will be collected and stored in a de-identified manner. Assessment and outcome questionnaires will be coded using random identification numbers, rather than (potentially) identifiable codes. Only the researchers will have access to the conversion key, which links the identification numbers to participants. Data and the conversion key will be stored in locked filing cabinets and in password-protected folders on password-protected computers.

\section{Statistical analysis plan}

Missing value analyses will be performed by the Little's missing completely at random (MCAR) test to determine whether values are (completely) missing at random. Main baseline characteristics will then be compared to determine if there are any relevant differences between participants with and without missing data. Characteristics will be compared both visually and statistically with independent sample t-test and Mann-Whitney U test. Multiple imputation methods will be performed on the predictors and outcomes with missing values by the
Multivariate Imputation by Chained Equations method with Predictive Mean Matching. The number of imputations will be related to the percentage of missing data. The association between the potential predictor variables and programme success will be evaluated using multivariable logistic regression analyses with backward Wald selection. In agreement with the TRIPOD statement, we use automatic backward Wald selection, but use 0.157 rather than 0.05 , to increase the likelihood that predictors are included in the model. We correct for optimism by internal validation using bootstrapping.

All assumptions (linearity between independent continuous variables, $\log$ odds and multicollinearity) will be checked before model building. Predictor variables will be entered as continuous variables (if there is a linear relation with the outcome).

How well the prognostic models fit the data will be determined with the Hosmer-Lemeshow test and the explained variance with the Nagelkerke R2. Discriminative ability of the two models will be assessed using the area under the receiver operating characteristic curve. An area under the curve of 0.5 indicates poor discrimination above chance, 0.7 fair discrimination, 0.8 acceptable discrimination, whereas an area under the curve of 1.0 indicates perfect discrimination. To correct for overfitting, the internal validity of the models will be assessed through bootstrapping techniques with 500 repetitions. If feasible, we will develop prognostic models based on analysis from those participants who complete all five sessions of UPLIFT and analysis from all participants who complete a minimum of three sessions of the programme.

\section{Patient and public involvement}

Patients were involved in the assessment of the burden of the intervention and extra time required to participate in the research, for example, the extra time needed to complete additional self-reported questionnaires and outcome measures. Patient feedback has also influenced the content and delivery modes of the intervention. We will invite patient and public representatives to assist us to develop our dissemination strategy to patient groups. Patient and public representatives were not involved in the formulation of the research question, study design or outcome measures.

\section{Ethics and dissemination}

The study is registered in the Australian New Zealand Clinical Trials Registry (ACTRN12618001525279). The results of the study will be published in peer-reviewed journals and disseminated at several conferences.

\section{DISCUSSION}

The present study will evaluate whether good and poor outcome to the UPLIFT programme can be accurately identified when considering baseline data from various psychosocial domains. There is a growing body of evidence indicating that patient beliefs and psychosocial factors, 
such as pain catastrophising, self-efficacy and patient expectations, are better predictors for the management of people with LBP than biomechanical and structural pathology. ${ }^{24} 51$ Patients with reduced physical function, psychological distress, negative feelings about their LBP and increased fear of movement, are more disabled by their LBP and are more likely to have a poor outcome. ${ }^{52}$ Evidence suggests an absence of clinically meaningful long-term effects of interventions and the trend towards increasing LBP disability and chronicity. ${ }^{24}$

People with LBP referred to specialist secondary healthcare services present with increased pain intensity, reduced function and higher rates of poor prognosis than patients presenting to primary healthcare services. ${ }^{15} 53$ Before reaching the secondary care service, the vast majority of patients with LBP have already failed a course of conservative treatment. ${ }^{10}$ Secondary healthcare clinicians are then faced with the challenge of providing time and cost-efficient, evidence-based interventions for this population group. ${ }^{53}$ A recent review highlighted that research aiming to reveal factors that identify responders in secondary healthcare is lacking. ${ }^{4}$ To date, there is no evidence that the prognostic variables identified in primary healthcare can be translated into secondary healthcare. ${ }^{4}$

The health service and patient outcomes may be further improved if good and poor outcome to the UPLIFT programme can be identified more accurately and more objectively at baseline. There is a need to improve the appropriate utilisation of the UPLIFT programme and evaluate and further improve patient outcomes following the programme. There is a recognised need to develop an easy-to-use screening tool at baseline that may help identify those patients likely to gain the most benefit from the UPLIFT programme and, conversely, those patients who are at risk of a poor outcome who may require alternative treatment approaches. This pragmatic study, which reflects existing clinical practice, aims to derive prognostic models to identify good and poor outcome to the UPLIFT programme. If valuable models can be developed, validation of the models in future studies will be essential before the models can be recommended in clinical practice. Furthermore, identification of prognostic factors may help in the future development of screening tools to help guide treatment direction.

\section{Author affiliations}

${ }^{1}$ Gold Coast University Hospital, Gold Coast Hospital and Health Service, Gold Coast, Australia

${ }^{2}$ School of Medical Science, Griffith University, Gold Coast, Australia

${ }^{3}$ Faculty of Health Sciences, The University of Sydney, Sydney, Australia

${ }^{4}$ School of Allied Health Sciences, Griffith University, Brisbane \& Gold Coast, Australia

${ }^{5}$ Allsports Physiotherapy and Sports Medicine Clinics, Healthia Limited, Brisbane,

Australia

${ }^{6}$ The Hopkins Centre, Menzies Health Institute Queensland, Griffith University, Brisbane \& Gold Coast, Australia

${ }^{7}$ Faculty of Behavioural and Movement Sciences, Vrije Universiteit Amsterdam, Amsterdam Movement Sciences, Amsterdam, The Netherlands
${ }^{8}$ Department of Clinical Psychology, Vrije Universiteit Amsterdam, Amsterdam, The Netherlands

Contributors Conceptualisation: KE, MWC, JK, JD, HWT. Acquisition of funding: MWC, KE. Development of intervention: JK, KC, JD, HWT. Development of protocol: MWC, KE, LB, HWT, JD, CM, GS-P, PC. Writing —original draft: KE, MWC, HWT, JD, GS-P. Writing - review and editing: the final version of the protocol was approved by all the authors.

Funding This work is supported by Gold Coast Hospital and Health Service and Gold Coast Hospital Foundation grant number SG0027.

Disclaimer The funding source has no role in study design, data collection, analysis and interpretation, in the writing of the report or in the decision to submit the paper for publication.

Competing interests None declared.

Patient consent for publication Not required.

Ethics approval The Human Research Ethics Committee of Gold Coast Health Service (HREC/18/QGC/41) and Griffith University (GU Ref No: 2018/408) approved the study proposal.

Provenance and peer review Not commissioned; externally peer reviewed.

Open access This is an open access article distributed in accordance with the Creative Commons Attribution Non Commercial (CC BY-NC 4.0) license, which permits others to distribute, remix, adapt, build upon this work non-commercially, and license their derivative works on different terms, provided the original work is properly cited, appropriate credit is given, any changes made indicated, and the use is non-commercial. See: http://creativecommons.org/licenses/by-nc/4.0/.

\section{REFERENCES}

1. Hartvigsen J, Hancock MJ, Kongsted A, et al. What low back pain is and why we need to pay attention. Lancet 2018;391.

2. Hoy D, March L, Brooks P, et al. The global burden of low back pain: estimates from the Global Burden of Disease 2010 study. Ann Rheum Dis 2014;73:968-74.

3. Freburger JK, Holmes GM, Agans RP, et al. The rising prevalence of chronic low back pain. Arch Intern Med 2009;169:251-8.

4. Karran EL, Traeger AC, McAuley JH, et al. The Value of Prognostic Screening for Patients With Low Back Pain in Secondary Care. $J$ Pain 2017;18:673-86.

5. Britt H, Miller GC, Charles J, et al. General practice activity in Australia 2000-01 to 2009-10: 10 year data tables. General practice series 2010;28:37-9.

6. Robarts S, Stratford P, Kennedy D, et al. Evaluation of an advancedpractice physiotherapist in triaging patients with lumbar spine pain: surgeon-physiotherapist level of agreement and patient satisfaction. Can J Surg 2017;60:266-72.

7. Foster NE, Anema JR, Cherkin D, et al. Prevention and treatment of low back pain: evidence, challenges, and promising directions. Lancet 2018;391.

8. Bernstein IA, Malik Q, Carville S, et al. Low back pain and sciatica: summary of NICE guidance. BMJ 2017;356:i6748.

9. Maher C, Underwood M, Buchbinder R. Non-specific low back pain. Lancet 2017:389:736-47.

10. Kamper SJ, Apeldoorn AT, Chiarotto A, et al. Multidisciplinary biopsychosocial rehabilitation for chronic low back pain. Cochrane Database Syst Rev 2014;9:CD000963.

11. Falla D, Hodges PW. Individualized Exercise Interventions for Spinal Pain. Exerc Sport Sci Rev 2017;45:105-15.

12. Hingorani $A D$, Windt $D A$, Riley RD, et al. Prognosis research strategy (PROGRESS) 4: stratified medicine research. BMJ 2013;346:e5793.

13. van der Windt DA, Dunn KM. Low back pain research-future directions. Best Pract Res Clin Rheumatol 2013;27:699-708.

14. Hill JC, Whitehurst DG, Lewis $M$, et al. Comparison of stratified primary care management for low back pain with current best practice (STarT Back): a randomised controlled trial. Lancet 2011;378:1560-71.

15. Mors $\varnothing \mathrm{L}$, Kent P, Albert HB, et al. Is the psychosocial profile of people with low back pain seeking care in Danish primary care different from those in secondary care? Man Ther 2013;18:54-9.

16. Foster NE, Mullis R, Hill JC, et al. Effect of stratified care for low back pain in family practice (IMPaCT Back): a prospective populationbased sequential comparison. Ann Fam Med 2014;12:102-11.

17. Unsgaard-Tøndel M, Kregnes IG, Nilsen TIL, et al. Risk classification of patients referred to secondary care for low back pain. BMC Musculoskelet Disord 2018;19:166. 
18. Koes BW, van Tulder M, Lin CW, et al. An updated overview of clinical guidelines for the management of non-specific low back pain in primary care. Eur Spine J 2010;19:2075-94.

19. Moseley LG, Butler DS. Explain Pain Supercharged. 1st edn. South Australia: Noigroup Publications, 2017.

20. Biggs JT. Teaching For Quality Learning At University. 4th edn. London: McGraw-Hill Education, 2011.

21. Hayden JA, van Tulder MW, Malmivaara A, et al. Exercise therapy for treatment of non-specific low back pain. Cochrane Database Syst Rev 2005:CD000335.

22. Smith BE, Littlewood C, May S. An update of stabilisation exercises for low back pain: a systematic review with meta-analysis. BMC Musculoskelet Disord 2014;15:416.

23. Qaseem A, Wilt TJ, McLean RM, et al. Noninvasive Treatments for Acute, Subacute, and Chronic Low Back Pain: A Clinical Practice Guideline From the American College of Physicians. Ann Intern Med 2017;166:514-30.

24. O'Sullivan P, Caneiro JP, O'Keeffe M, et al. Unraveling the Complexity of Low Back Pain. J Orthop Sports Phys Ther 2016;46:932-7.

25. Testa M, Rossettini G. Enhance placebo, avoid nocebo: How contextual factors affect physiotherapy outcomes. Man Ther 2016;24:65-74.

26. Wilson CA, Roffey DM, Chow D, et al. A systematic review of preoperative predictors for postoperative clinical outcomes following lumbar discectomy. Spine J 2016;16:1413-22.

27. Cook CE, Arnold PM, Passias PG, et al. Association for Collaborative Spine Research (ACSR). Predictors of pain and disability outcomes in one thousand, one hundred and eight patients who underwent lumbar discectomy surgery. Int Orthop 2015;39:2143-51.

28. Waddell G, Newton M, Henderson I, et al. A Fear-Avoidance Beliefs Questionnaire (FABQ) and the role of fear-avoidance beliefs in chronic low back pain and disability. Pain 1993;52:157-68.

29. Sleijser-Koehorst MLS, Bijker L, Cuijpers P, et al. Preferred selfadministered questionnaires to assess fear of movement, coping, self-efficacy, and catastrophizing in patients with musculoskeletal pain-A modified Delphi study. Pain 2019;160:600-606.

30. Nicholas MK. The pain self-efficacy questionnaire: Taking pain into account. Eur J Pain 2007;11:153-63.

31. Dima A, Lewith GT, Little P, et al. Patients' treatment beliefs in low back pain: development and validation of a questionnaire in primary care. Pain 2015:156:1489-500.

32. Sullivan MJ, Adams H, Horan S, et al. The role of perceived injustice in the experience of chronic pain and disability: scale development and validation. J Occup Rehabil 2008;18:249-61.

33. Osman A, Barrios FX, Kopper BA, et al. Factor structure, reliability, and validity of the Pain Catastrophizing Scale. J Behav Med 1997;20:589-605.

34. Osman A, Barrios FX, Gutierrez PM, et al. The Pain Catastrophizing Scale: further psychometric evaluation with adult samples. J Behav Med 2000;23:351-65.

35. Lovibond PF, Lovibond SH. The structure of negative emotional states: comparison of the Depression Anxiety Stress Scales (DASS) with the Beck Depression and Anxiety Inventories. Behav Res Ther 1995;33:335-43.
36. Bijker L, Sleijser-Koehorst M, Coppieters M, et al. Preferred selfadministered questionnaires to assess depression, anxiety and somatization in people with musculoskeletal pain - A modified Delphi study. Manuscript submitted for publication, 2018.

37. Fairbank JC, Pynsent PB. The Oswestry Disability Index. Spine 2000;25:2940-53.

38. Vianin M. Psychometric properties and clinical usefulness of the Oswestry Disability Index. J Chiropr Med 2008;7:161-3.

39. Wand BM, O'Connell NE, Di Pietro F, et al. Managing chronic nonspecific low back pain with a sensorimotor retraining approach: exploratory multiple-baseline study of 3 participants. Phys Ther 2011;91:535-46.

40. Cleeland C. The brief pain inventory user guide 2009. Cleeland Charles S, Houston, TX Google Scholar, 2014

41. Ware JE. SF-36 health survey update. Spine 2000;25:3130-9.

42. Ruta DA, Abdalla MI, Garratt AM, et al. SF 36 health survey questionnaire: I. Reliability in two patient based studies. Qual Health Care 1994;3:180-5.

43. Ware JE, Sherbourne CD. The MOS 36-item short-form health survey (SF-36). I. Conceptual framework and item selection. Med Care 1992;30:473-83.

44. Lee RM, Robbins SB. Measuring belongingness: The Social Connectedness and the Social Assurance scales. J Couns Psychol 1995;42:232-41.

45. Dworkin RH, Turk DC, Farrar JT, et al. Core outcome measures for chronic pain clinical trials: IMMPACT recommendations. Pain 2005;113:9-19.

46. Kamper SJ, Maher CG, Mackay G. Global rating of change scales: a review of strengths and weaknesses and considerations for design. $J$ Man Manip Ther 2009;17:163-70.

47. Norton KI, Norton L. Pre-exercise screening: Guide to the Australian adult pre-exercise screening system: Exercise and Sports Science Australia, 2011

48. Toh I, Chong HC, Suet-Ching Liaw J, et al. Evaluation of the STarT Back Screening Tool for Prediction of Low Back Pain Intensity in an Outpatient Physical Therapy Setting. J Orthop Sports Phys Ther 2017:47:261-7

49. Gellhorn AC, Suri P, Rundell SD, et al. Lumbar Muscle CrossSectional Areas Do Not Predict Clinical Outcomes in Adults With Spinal Stenosis: A Longitudinal Study. PM\&R 2017;9:545-55.

50. Azimi P, Benzel EC, Shahzadi S, et al. The prediction of successful surgery outcome in lumbar disc herniation based on artificial neural networks. J Neurosurg Sci 2016;60:173-7.

51. Hall AM, Ferreira PH, Maher CG, et al. The influence of the therapistpatient relationship on treatment outcome in physical rehabilitation: a systematic review. Phys Ther 2010;90:1099-110.

52. Moseley GL, Butler DS. Fifteen years of explaining pain: the past, present, and future. J Pain 2015;16:807-13.

53. Karran EL, Hillier SL, Yau YH, et al. A quasi-randomised, controlled, feasibility trial of GLITtER (Green Light Imaging Interpretation to Enhance Recovery)-a psychoeducational intervention for adults with low back pain attending secondary care. PeerJ 2018;6:e4301.

54. Moseley LG. Painful Yarns. Metaphors and Stories to Help Understand the Biology of Pain. Canberra, Australia: Dancing Giraffe Press, 2007. 